\title{
CRÓNICA PARLAMENTARIA DEL CONGRESO DE LOS DIPUTADOS
}

\author{
AURORA GUTIÉRREZ NOGUEROLES
}

Profesora Asociada de Derecho Constitucional UNED 


\section{SUMARIO}

I. Actividad legislativa. 1. Leyes. 1.1. Leyes orgánicas. 1.2. Leyes ordinarias. 2. Decretos-leyes. 3. Proposiciones de Ley. 


\title{
CRÓNICA PARLAMENTARIA DEL CONGRESO DE LOS DIPUTADOS
}

\author{
POR \\ AURORA GUTIÉRREZ NOGUEROLES \\ Profesora Asociada de Derecho Constitucional \\ UNED
}

Constituye objeto de nuestra habitual crónica parlamentaria la actividad desarrollada por el Congreso de los Diputados durante el período de enero a diciembre de 1998, ambos inclusive, meses que corresponden al quinto y sexto períodos de sesiones de la actual VI Legislatura. Hay que reiterar, de nuevo, que nos limitamos a destacar, dentro de la producción total de la Cámara, sólo aquella actividad que así lo merece, desde nuestra particular visión. Para una mayor claridad en la exposición, la actividad del Congreso aparece, como en ocasiones anteriores, desglosada en los dos apartados habituales: actividad legislativa y actividad no legislativa.

\section{ACTIVIDAD LEGISLATIVA}

\section{LEYES}

\subsection{Leyes orgánicas}

Durante los dos períodos de sesiones aludidos, han sido aprobadas once leyes orgánicas, que son las relacionadas a continuación: 


\section{Materia}

Reforma de la L.O. 4/1982, de 9 de junio, del Estatuto de Autonomía para la Región de Murcia.

Modificación del Código Penal y de la Ley de Enjuiciamiento Criminal.

Modificación de la Ley Orgánica del Régimen Electoral General.

Cooperación con el Tribunal Internacional para Ruanda.

Reforma de la L.O. 3/1983, de 25 de febrero, de Estatuto de Autonomía de la Comunidad de Madrid.

Reforma de la Ley Orgánica del Poder Judicial.

Modificación de la L.O. 10/1995, de 23 de noviembre, del Código Penal, por la que se suprimen las penas de prisión y multa para los supuestos de incumplimiento del servicio militar obligatorio y prestación social sustitutoria y se rebajan las penas de inhabilitación para dichos supuestos.

Régimen Disciplinario de las Fuerzas Armadas.

Autorización de la ratificación por España del Tratado de Amsterdam, por el que se modifican el Tratado de la Unión Europea, los Tratados constitutivos de las Comunidades Europeas y determinados actos conexos.

Complementaria de la ley sobre introducción del euro.

Reforma de la L.O. 8/1981, de 30 de diciembre, del Estatuto de Autonomía para Cantabria.
$005 \quad 07 / 07 / 98 \quad 162$

$006 \quad 13 / 07 / 98$ 167

$008 \quad 02 / 12 / 98 \quad 289$

$02 / 12 / 98 \quad 289$

$010 \quad 17 / 12 / 98$ 302

N. ${ }^{\circ}$ Ley Fecha Ley BOE

$002 \quad 15 / 06 / 98 \quad 143$

$003 \quad 15 / 06 / 98 \quad 143$

$004 \quad 01 / 07 / 98 \quad 157$

$007 \quad 05 / 10 / 98 \quad 239$

16/12/98 301

$\begin{array}{lll}011 \quad 30 / 12 / 98 & 313\end{array}$ 


\subsection{Leyes ordinarias}

Durante los períodos de sesiones analizados, han sido aprobadas cincuenta leyes ordinarias, de las que podemos destacar las siguientes:

\begin{tabular}{ccc}
\hline Materia & N. Ley Fecha Ley & BOE \\
\hline
\end{tabular}

Derechos y garantías de los contribuyentes.

$001 \quad 26 / 02 / 98 \quad 50$

Cambio de denominación de las provincias de A Coruña y Ourense.

$002 \quad 03 / 03 / 98 \quad 54$

Creación del Consejo General de Colegios

Oficiales de Podólogos.

$003 \quad 03 / 03 / 98 \quad 54$

Establecimiento del régimen sancionador previsto en el Reglamento (CE) 3093/1994, del Consejo, de 15 de diciembre, relativo a sustancias que agotan la capa de ozono.

$004 \quad 03 / 03 / 98 \quad 54$ Incorporación al Derecho español de la Directiva 96/9/CE, del Parlamento Europeo y del Consejo, de 11 de marzo de 1996, sobre la protección jurídica de las bases de datos.

Régimen del suelo y valoraciones.

$005 \quad 06 / 03 / 98 \quad 57$

Condiciones generales de la contratación. $006 \quad 13 / 04 / 98 \quad 89$

Ampliación del concepto de familia numerosa.

$007 \quad 13 / 04 / 98 \quad 89$

Modificación de la Ley 37/1992, de 28 de diciembre, del Impuesto sobre el Valor Añadido.

$009 \quad 21 / 04 / 98 \quad 96$

Residuos.

$010 \quad 21 / 04 / 98 \quad 96$

General de Telecomunicaciones.

$011 \quad 24 / 04 / 98 \quad 99$

Modificación de la Ley 13/1994, de 1 de junio, de autonomía del Banco de España.

$012 \quad 28 / 04 / 98 \quad 102$

Ordenación del mercado de tabacos

y normativa tributaria.

$013 \quad 04 / 05 / 98 \quad 107$

Establecimiento del régimen de control para protección de los recursos pesqueros.

$014 \quad 01 / 06 / 98 \quad 131$ 


\section{Materia}

N. ${ }^{\circ}$ Ley Fecha Ley BOE

Modificación parcial de la Ley 36/1994, de 23 de diciembre, relativa a la restitución de bienes culturales que hayan salido de forma ilegal del territorio de un Estado miembro de la Unión Europea.

Modificación del Convenio Económico entre el Estado y la Comunidad Foral de Navarra.

$019 \quad 15 / 06 / 98$ 143

Reforma del régimen jurídico y fiscal de las instituciones de inversión colectiva de naturaleza inmobiliaria y sobre cesión de determinados derechos de crédito de la Administración General del Estado. Creación del Consejo General de Colegios de Fisioterapeutas.

$\begin{array}{lll}020 & 01 / 07 / 98 & 157\end{array}$

$021 \quad 01 / 07 / 98 \quad 157$

Reguladora de la objeción de conciencia y prestación social sustitutoria.

$\begin{array}{lll}022 & 07 / 07 / 98 & 161\end{array}$

Cooperación internacional para el desarrollo.

$023 \quad 07 / 07 / 98 \quad 162$

Servicio postal universal y de liberalización de los servicios postales.

$\begin{array}{lll}024 & 13 / 07 / 98 & 167\end{array}$

Modificación del régimen legal de las tasas estatales y locales y de reordenación de las prestaciones patrimoniales de carácter público.

$\begin{array}{lll}025 & 13 / 07 / 98 & 167\end{array}$

Modificación de la Ley 38/1988, de 28 de diciembre, de demarcación $y$ de planta judicial.

Sanciones aplicables a las infracciones de las normas establecidas en el Reglamento (CE) n. ${ }^{\circ} 2271 / 96$, de 22 de noviembre, del Consejo, relativo a la protección frente a la aplicación extraterritorial de la legislación de un pais tercero.

Venta a plazos de bienes muebles.

Reguladora de la Jurisdicción Contenciosoadministrativa.

Régimen especial de las llles Balears. 


Materia
Prohibición total de minas antipersonal
y armas de efecto similar.

Sector de hidrocarburos.

Creación del Colegio Oficial de Pilotos de la Aviación Comercial.

Modificación del artículo 14, apartados $1 .^{\circ}$ y $3 .^{\circ}$, de la Ley de Enjuiciamiento Criminal.

Reforma de la Ley 24/1988, de 28 de julio, del Mercado de Valores.

$037 \quad 16 / 11 / 98$

275

Modificación de la composición de la Comisión Nacional de Ayuda y Asistencia a las Víctimas de Delitos Violentos y contra la Libertad Sexual. Impuesto sobre la Renta de las Personas Físicas $y$ otras normas tributarias.

Renta de no residentes y normas tributarias.

Derechos de aprovechamiento por turno de bienes inmuebles de uso turístico y normas tributarias.

038

$27 / 11 / 98$

285

$040 \quad 09 / 12 / 98 \quad 295$

$041 \quad 09 / 12 / 98 \quad 295$

(y 301)

Restitución o compensación a los partidos políticos de bienes y derechos incautados en aplicación de la normativa sobre responsabilidades políticas del período 1936-1939.

$043 \quad 15 / 12 / 98 \quad 300$

Planta y organización territorial de la jurisdicción militar.

Introducción del euro.

Reglas para el reconocimiento de la jubilación anticipada de la Seguridad Social, en determinados casos especiales.

$044 \quad 15 / 12 / 98 \quad 300$

$046 \quad 17 / 12 / 98 \quad 302$

Procedimientos de contratación en los sectores del agua, la energía, los transportes $y$ las telecomunicaciones, por la que se incorporan al ordenamiento jurídico español las Directivas 93/38/CEE y 92/13/CEE. $048 \quad 30 / 12 / 98 \quad 313$ 


\begin{tabular}{lccc}
\hline \multicolumn{1}{c}{ Materia } & $N .{ }^{\circ}$ Ley & Fecha Ley & $B O E$ \\
\hline $\begin{array}{l}\text { Presupuestos Generales del Estado } \\
\text { para 1999. }\end{array}$ & 049 & $30 / 12 / 98$ & 313 \\
$\begin{array}{l}\text { Medidas fiscales, administrativas } \\
\text { y del orden social. }\end{array}$ & 050 & $30 / 12 / 98$ & 313 \\
\hline
\end{tabular}

\section{DECRETOS-LEYES}

En el transcurso del período que se estudia, han sido convalidados veinte Reales Decretos-leyes, de los que cabe destacar los siguientes:

\begin{tabular}{lll}
\hline Materia & $N{ }^{\circ} D-L$ Fecha $D-L$ & $B O E$ \\
\hline
\end{tabular}

Infraestructuras comunes en los edificios para el acceso a los servicios de telecomunicación.

$001 \quad 27 / 02 / 98 \quad 51$

Adopción de medidas urgentes para reparar los daños causados por las inundaciones y temporales acaecidos entre noviembre de 1997 y febrero de 1998.

Establecimiento de las retribuciones de los magistrados del Tribunal Supremo, en cumplimiento de lo dispuesto en el artículo 404 bis de la Ley Orgánica 6/1985, de 1 de julio, del Poder Judicial, añadido por la Ley Orgánica 5/1997, de 4 de diciembre, y aprobación del correspondiente suplemento de crédito, por un importe total de 609.680 .793 pesetas, para su financiación.

$003 \quad 08 / 05 / 98 \quad 111$

Reglas para el reconocimiento de la jubilación anticipada del sistema de la Seguridad Social, en determinados casos especiales.

$005 \quad 29 / 05 / 98$ 129

Transformación de la Empresa Nacional de Transportes de Viajeros por Carretera (ENATCAR) en una sociedad anónima.

Modificación de la Ley 19/1994, de 6 de julio, de Modificación del Régimen Económico y Fiscal de Canarias. 


\section{Materia}

$N .^{\circ} D-L$ Fecha $D-L \quad B O E$

Medidas urgentes en materia de propiedad industrial.

$008 \quad 31 / 07 / 98$

194

Aprobación y declaración de interés general de determinadas obras hidráulicas.

$009 \quad 28 / 08 / 98$

207

Regulación de las bonificaciones de cuotas a la Seguridad Social de los contratos de interinidad celebrados con personas desempleadas para sustituir a trabajadores durante los períodos de descanso por maternidad, adopción y acogimiento.

$011 \quad 04 / 09 / 98 \quad 213$

Adhesión de España a diversos Acuerdos del Fondo Monetario Internacional.

$014 \quad 09 / 10 / 98 \quad 243$

Medidas urgentes para la mejora del mercado de trabajo en relación con el trabajo a tiempo parcial y el fomento de su estabilidad.

$015 \quad 27 / 11 / 98 \quad 285$

Financiación del Acuerdo Interprofesional sobre formación continua en la Comunidad Autónoma del País Vasco.

\section{PROPOSICIONES DE LEY}

De las ciento veintiuna proposiciones de ley que han sido presentadas a lo largo de los dos periodos de sesiones que estudiamos, ciento once lo fueron a iniciativa de los diversos Grupos parlamentarios del Congreso, ocho a iniciativa de las Asambleas legislativas de las Comunidades Autónomas (concretamente, de Asturias, Canarias, Cantabria, Castilla y León, Cataluña, Extremadura, Islas Baleares y La Rioja) y las dos restantes por iniciativa del Senado. Las materias sobre las que versaban estas dos últimas proposiciones de ley eran, respectivamente, la adaptación de las normas de circulación a la práctica del ciclismo (la presentada el 9 de octubre) y la modificación de los artículos 19 y 240 de la Ley Orgánica 6/1985, de 1 de julio, del Poder Judicial (la presentada el 12 de febrero). Ambas fueron tomadas en consideración por el Pleno del Congreso.

Debe igualmente significarse que una de las proposiciones de ley fue presentada, en fecha 4 de febrero, por la iniciativa conjunta de tres Grupos parlamentarios (Catalán, Coalición Canaria y Popular), siendo 
su objeto la supresión de las penas de prisión y multa para los supuestos de no cumplimiento del servicio militar obligatorio y prestación social sustitutoria, así como la rebaja de las penas de inhabilitación para dichos supuestos. Una vez tomada en consideración por el Pleno del Congreso y tras sus correspondientes trámite y aprobación, se convertiría en la L.O. 7/1998, de 5 de octubre, que modificaba el Código Penal en los mencionados extremos.

Dejando al margen las dos proposiciones de ley, antes aludidas, de iniciativa de la Cámara Alta, el cuadro estadístico que podría configurarse con las demás sería el siguiente:

\begin{tabular}{lcccccccc}
\hline \multicolumn{1}{c}{$\begin{array}{c}\text { Proposiciones } \\
\text { de } \\
\text { Ley }\end{array}$} & Cat & Ccan & IU-IC & Mixto & Pop. & Soc. & Conjunta & $\begin{array}{c}\text { Asambleas } \\
\text { Legislativas }\end{array}$ \\
\hline Autonómicas
\end{tabular}

A continuación, detallamos las setenta y seis proposiciones de ley que fueron tomadas en consideración por el Pleno del Congreso, desglosándolas en función del Grupo parlamentario o Asamblea legislativa proponente:

\section{a) Del Grupo Catalán}

- (Orgánica) Medidas para favorecer una mayor protección e integración de los inmigrantes (fecha de presentación: 06/03/98).

- Modificación de la Ley 11/98, de 24 de abril, General de Telecomunicaciones, por la que se otorga a Internet la consideración de servicio universal (fecha de presentación: 10/12/98).

\section{b) Del Grupo Coalición Canaria}

- (Orgánica) Modificación del artículo 75 de la Ley Orgánica 5/1985, de 19 de junio, del Régimen Electoral General. 


\section{c) Del Grupo Izquierda Unida-IC}

- (Orgánica) Reforma de la Ley Orgánica 7/1985, de 1 de julio, sobre derechos y libertades de los extranjeros en España.

- (Orgánica) Reconocimiento de los derechos sufragio activo y pasivo a determinados ciudadanos extranjeros.

- Modificación del Código Civil y de la Ley de 8 de junio de 1957 del Registro Civil, en materia de inscripción de apellidos.

- Participación de las Comunidades Autónomas en los Consejos de Administración y órganos de dirección de los organismos económicos, instituciones financieras y empresas públicas de titularidad estatal.

- Creación de un Fondo de Compensación para indemnizar a las Corporaciones Locales que se han visto afectadas por las sentencias firmes de los Tribunales, en relación con la declaración de inconstitucionalidad de algunos preceptos del Real Decreto Legislativo 1/1992, de 26 de junio, por el que se aprueba el Texto Refundido de la Ley sobre el Régimen del Suelo y Ordenación Urbana.

- Cotización al Régimen Especial de los Trabajadores del Mar.

- Modificación parcial de la Ley de Arrendamientos Urbanos.

- (Orgánica) Reconocimiento como tribunal consuetudinario y tradicional del Consejo de Hombres Buenos de Murcia.

- (Orgánica) Reforma de la Ley Orgánica 3/1981, de 6 de abril, del Defensor del Pueblo, para su adecuación a la realidad plurilingüe del Estado.

- Modificación de la Ley de 8 de junio de 1957, reguladora del Registro Civil.

- (Orgánica) Modificación de la Ley Orgánica 11/1983, de 25 de agosto, de Reforma Universitaria.

- Consideración como cotizado a la Seguridad Social del período de excedencia por cuidado de menor de tres años y financiación de su coste a cargo de los Presupuestos Generales del Estado.

- Procedimiento preferente y sumario de las demandas para el reconocimiento de los permisos por lactancia o guarda legal.

- Medidas para facilitar la inserción familiar de los menores en los supuestos de acogimiento familiar y adopción.

- Concesión del subsidio de desempleo y de garantías de integración sociolaboral para los delincuentes toxicómanos que hayan visto re- 
mitida la ejecución de su pena de conformidad con lo previsto en la legislación penal.

- (Orgánica) Reconocimiento de la cooficialidad de las lenguas oficiales de Comunidades Autónomas en el procedimiento para el ejercicio de la iniciativa legislativa popular.

- (Orgánica) Modificación de la Ley Orgánica 5/1985, de 19 de junio, de Régimen Electoral General, en materia de uso de lenguas oficiales ante la Administración electoral.

- Modificación de los requisitos temporales para el cobro de prestaciones no contributivas al colectivo de emigrantes españoles retornados.

- Modificación de la Ley de Demarcación y Planta Judicial.

- Modificación del Código Civil en materia de adquisición y recuperación de la nacionalidad.

- (Orgánica) Modificación del artículo 14.4 de la Ley Orgánica 13/1991, de $\mathbf{2 0}$ de diciembre, del Servicio Militar.

- Modificación del Código Civil en materia del proceso de separación y divorcio cuando tengan como precedentes malos tratos entre los cónyuges.

\section{d) Del Grupo Mixto}

- Declaración como cooficiales de las denominaciones de Alacant, Castelló y València para las provincias que integran la Comunidad Valenciana.

- Tramitación preferente y urgente de las demandas judiciales para el reconocimiento de los derechos previstos en los apartados 4 y 5 del artículo 37 del Estatuto de los Trabajadores.

- (Orgánica) Creación de la provincia de Cataluña.

- (Orgánica) Modificación de algunos artículos de la Ley Orgánica 13/1991, de 20 de diciembre, del Servicio Militar y del Reglamento de Reclutamiento, aprobado por el Real Decreto 1107/1993, de 9 de julio, sobre la participación de los Ayuntamientos en el reclutamiento.

- (Orgánica) Reforma de la Ley Orgánica 7/1985, de 1 de julio, sobre derechos y libertades de los extranjeros en España.

- Cotización en el régimen especial de trabajadores del mar.

- Modificación de la Ley 27/1992, de 24 de noviembre, de Puertos del Estado y de la Marina Mercante. 
- (Orgánica) Modificación de la Ley Orgánica 3/1981, del Defensor del Pueblo.

- (Orgánica) Modificación de la Ley Orgánica 10/1995, de 23 de noviembre, del Código Penal y de la Ley de Enjuiciamiento Criminal, aprobada por Real Decreto de 14 de septiembre de 1882, en lo relativo a los delitos de lesiones causadas al cónyuge y convivientes.

- Modificación de la Ley de Demarcación y Planta Judicial para la extensión de la jurisdicción del Juzgado de lo Social de Mieres a los partidos judiciales de Lena, Langreo y Laviana.

- (Orgánica) Reconocimiento de la cooficialidad de las lenguas oficiales de Comunidades Autónomas en el procedimiento para el ejercicio de la iniciativa legislativa popular.

- (Orgánica) Equiparación del tratamiento penal de todas las conductas de insumisión al servicio militar.

\section{e) Del Grupo Popular}

- Creación del Comité de Medios Audiovisuales.

- Obligaciones de las empresas de transportes en relación con el régimen de viajeros no pertenencientes a países de la Unión Europea.

- Modificación del Código Civil en materia de inscripción de apellidos.

- Modificación del artículo 9, apartado 5, del Código Civil.

- Modificación de la Ley 7/1985, de 2 de abril, reguladora de las Bases del Régimen Local, en materia de financiación de las actividades de los grupos políticos constituidos en las entidades locales.

- Ampliación del permiso laboral en caso de adopción.

- Modificación del artículo 20.1 del Código Civil.

- Elaboración de un texto refundido de la Ley de Seguridad Social de los Funcionarios Civiles del Estado.

- Establecimiento de nuevos tipos de infracciones en materia de seguridad ciudadana.

- Creación del Colegio Profesional de Pedagogos.

- Reforma del Código Civil en materia de procesos de separación y divorcio, cuando se deriven o tengan como precedentes malos tratos entre los cónyuges. 
- Modificación del artículo 53 de la Ley del Registro Civil, para posibilitar que hijos e hijas puedan llevar como primer apellido el materno desde su nacimiento, si así lo deciden sus progeniotores.

- Creación del Consejo Superior de los Medios Audiovisuales.

- Creación de un Fondo de Compensación a favor de los Ayuntamientos afectados por sentencias de los Tribunales Superiores de Justicia sobre el Real Decreto Legislativo 1/1992, por el que se aprueba el Texto Refundido de la Ley sobre Régimen del Suelo y Ordenación Urbana.

- Modificación del Impuesto sobre la Renta de las Personas Físicas (IRPF), para el cumplimiento de lo dispuesto en la Disposición Final Cuarta de la Ley 29/1994, de 24 de noviembre, de Arrendamientos Urbanos.

- Modificación de la Ley 7/1985, de 2 de abril, reguladora de las Bases del Régimen Local, en materia de financiación de las actividades de los grupos políticos constituidos en las entidades locales.

- Consideración como cotizado a la Seguridad Social del período de reserva del puesto de trabajo por cuidado de menor de tres años y financiación de su coste a cargo de los Presupuestos Generales del Estado.

- Consideración, a efectos de prórroga para el servicio militar obligatorio y la prestación social sustitutoria, de que el interesado tenga un contrato de formación, de prácticas o de cualquier modalidad de contratación temporal.

- (Orgánica) Modificación de la Ley Orgánica 6/1985, de 1 de julio, del Poder Judicial, por la que se reconoce el carácter de tribunal tradicional y consuetudinario al denominado Consejo de Hombres Buenos de Murcia.

- (Orgánica) Responsabilidad penal del menor.

- Modificación del artículo $4 .^{\circ}$ de la Ley 3/1981, de 25 de marzo, de creación del Parque Nacional de Garajonay (Isla de La Gomera).

- Concesión de subsidio de desempleo y de garantías de integración socio-laboral para los delincuentes toxicómanos que hayan visto suspendida la ejecución de su pena, de conformidad con lo previsto en el Código Penal. 
- Deducción por minusvalía en el Impuesto sobre la Renta de las Personas Físicas.

- Mejora de la inserción social de los menores en los supuestos de adopción y acogimiento familiar.

- Modificación de normas relativas al régimen de concesión y explotación de autopistas de peaje.

- Uso de lenguas oficiales ante la Administración electoral.

- (Orgánica) Uso de lenguas oficiales en el procedimiento para el ejercicio de la iniciativa legislativa popular.

- Modificación parcial del Anexo IX de la Ley 38/1988, de 28 de diciembre, de Demarcación y Planta Judicial, en lo que afecta a la delimitación de la demarcación del Juzgado de lo Social de Mieres.

- Modificación de la Ley 14/1994, de 1 de junio, por la que se regulan las empresas de trabajo temporal.

- (Orgánica) Modificación de la Ley Orgánica 5/1985, de 19 de junio, del Régimen Electoral General.

g) Conjunta de los Grupos Catalán, Coalición Canaria y Popular

- (Orgánica) Supresión de las penas de prisión y multa para los supuestos de no cumplimiento del servicio militar obligatorio y prestación militar sustitutoria, y rebaja de las penas de inhabilitación para dichos supuestos.

h) De la Junta General del Principado de Asturias

- Reforma del Estatuto de Autonomía del Principado de Asturias.

i) Del Parlamento de Canarias

- Modificación del artículo 6.1 de la Ley 19/1994, de 6 de julio, de modificación del régimen económico y fiscal de Canarias.

j) De la Asamblea Regional de Cantabria

- Reforma del Estatuto de Autonomía de Cantabria. 
k) De las Cortes de Castilla y León

- Reforma del Estatuto de Autonomía de Castilla y León.

I) Del Parlamento de Cataluña

- Uso de las lenguas oficiales en la emisión de billetes y en la acuñación de monedas del euro.

m) De la Asamblea de Extremadura

- Reforma del Estatuto de Autonomía de Extremadura.

n) Del Parlamento de Illes Balears

- Reforma del Estatuto de Autonomía de las Islas Baleares.

n) De la Diputación General de La Rioja

- Reforma del Estatuto de Autonomía de La Rioja. 